\title{
Optical pumping of stored atomic ions (*)
}

\author{
D. J. Wineland, W. M. Itano, J. C. Bergquist, J. J. Bollinger \\ and J. D. Prestage
}

Time and Frequency Division, National Bureau of Standards, Boulder, Colorado 80303, U.S.A.

\begin{abstract}
Résumé. - Ce texte discute les expériences de pompage optique sur des ions atomiques confinés dans des pièges électromagnétiques. Du fait de la faible relaxation et des déplacements d'énergie très petits des ions confinés, on peut obtenir une très haute résolution et une très grande précision dans les expériences de pompage optique associé à la double résonance. Dans la ligne de l'idée de Kastler de "lumino-réfrigération » (1950), l'énergie cinétique des niveaux des ions confinés peut être pompée optiquement. Cette technique, appelée refroidissement laser, réduit sensiblement les déplacements de fréquence Doppler dans les spectres.
\end{abstract}

\begin{abstract}
Optical pumping experiments on atomic ions which are stored in electromagnetic "traps» are discussed. Weak relaxation and extremely small energy shifts of the stored ions lead to very high resolution and accuracy in optical pumpingdouble resonance experiments. In the same spirit of Kastler's proposal for « lumino refrigeration " (1950), the kinetic energy levels of stored ions can be optically pumped. This technique, which has been called laser cooling, significantly reduces Doppler frequency shifts in the spectra.
\end{abstract}

\section{Introduction.}

For more than thirty years, the technique of optical pumping, as originally proposed by Alfred Kastler [1], has provided information about atomic structure, atom-atom interactions, and the interaction of atoms with external radiation. This technique continues today as one of the primary methods used in atomic physics. Moreover, it is a tribute to Kastler's foresight that some of his ideas have only recently been realized - for example, the relatively new technique of laser cooling is essentially contained in Kastler's idea of "lumino-refrigeration " [1].

Experiments on optical pumping of ions have not been as extensive as those on neutral atoms partly due to the difficulty of producing and maintaining sufficient ion populations and partly due to the difficulty of producing the required optical radiation which for positive ions is usually in the ultraviolet region of the spectrum. The subject of optically pumped ions was reviewed by Weber [2] in 1977; most of the experiments reported there used buffer gas cells to hold the ions and gas discharges as sources of radiation.

Since that time, a number of experiments using electromagnetic ion "traps" and laser sources for optical pumping have been carried out. In this paper we will restrict our attention to the unique features of the traps for optical pumping experiments; the basic methods of trapping have been discussed elsewhere [3, 4].

${ }^{*}$ ) Work of the U.S. Government; not subject to U.S. copyright. 


\section{Properties of ion traps for optical pumping experiments.}

2.1 ReLAXATION. - Often in ion trap experiments, ions are stored under ultra-high vacuum conditions $\left(p \lesssim 10^{-8} \mathrm{~Pa}[5]\right.$; neutral density $n \lesssim 2.7 \times 10^{6} / \mathrm{cm}^{3}$ at room temperature) for long periods of time (hours are not uncommon). As opposed to buffer gas cell experiments, this suggests that ion-neutral relaxation processes such as electron spin relaxation are extremely weak. As an example, suppose that ionneutral relaxation proceeds at a typical Langevin rate for orbiting type collisions [6] : $k \cong 2 \times 10^{-9} \mathrm{~cm}^{3} / \mathrm{s}$. For a pressure of $10^{-8} \mathrm{~Pa}$ at room temperature, the relaxation time constant is given by $\tau \cong(k n)^{-1} \cong 190 \mathrm{~s}$. Using cryogenic vacuum systems, this relaxation time could be made much longer.

Of course in samples of many trapped ions, the ions " see » or interact with each other through the Coulomb interaction; this might lead to relaxation in various ways. For example, electron spin relaxation in ions with ${ }^{2} \mathrm{~S}_{1 / 2}$ ground states could be driven by the relativistic magnetic fields $\mathbf{B}_{\mathrm{c}}=\mathbf{v} \times \mathbf{E}_{\mathrm{c}} / c$, produced in ion-ion collisions. To estimate this effect, assume $B_{\mathrm{c}}$ has a magnitude equal to that given by the above expression where $\mathbf{E}_{\mathrm{c}}$ is the electric field at the distance of closest approach $r$ (here assumed to be the impact parameter) and a duration given by $2 r / v$ where $v$ is the magnitude of the root mean square velocity between colliding ions. Then the spin precession angle for a single ion-ion collision is given by $\Delta \theta=\left(4 \pi \mu_{\mathrm{B}} B_{\mathrm{c}} / h\right) .(2 \mathrm{r} / \mathrm{v})$ where $\mu_{\mathbf{B}}$ is the Bohr magneton and $h$ is Planck's constant. For ions of mass $100 \mathrm{u}$ (atomic mass units) at room temperature, the mean precession angle averaged over all distances of closest approach (from the minimum distance of closest approach, $d_{0}=q^{2} / 3 k_{\mathrm{B}} T$, up to the mean spacing between ions, $n^{-1 / 3}$, where $q$ is the ion charge, $k_{\mathrm{B}}$ is Boltzmann's constant and $n$ is the ion density in $\mathrm{cm}^{-3}$ ) is $\langle\Delta \theta\rangle \cong 1 \times 10^{-12} n^{1 / 3}$. We would expect the angle to increase from multiple collisions in a random walk fashion so that $\mathrm{d} \theta^{2} / \mathrm{d} t \cong\langle\Delta \theta\rangle^{2} v n^{1 / 3} \cong 5 \times 10^{-20} n$. Even for ion densities of $n \cong 10^{8} \mathrm{~cm}^{-3}$, such relaxation is negligible.

Relaxation might also occur in a similar way via electric dipole transitions - the rate would be approximately $(c / v)^{2}\left(q a_{0} / 2 \mu_{\mathrm{B}}\right)^{2}$ times faster or $\mathrm{d} \theta^{2} / \mathrm{d} t \cong 5 \times 10^{-4} n$ ( $a_{0}$ is the Bohr radius). However these calculations are valid only when the transition frequencies of interest are small compared to the inverse of the time of duration of the collision. If the transition frequencies are higher, then the states tend to adiabatically adjust during the collision and no relaxation occurs. Another way of saying this is that the spectrum of the $\mathbf{E}$ and $\mathbf{B}$ fields during the collision does not have components much above a frequency $v / r$. For room temperature ions $(M=100 \mathrm{u})$, even at the minimum distance of closest approach this cutoff frequency is around $20 \mathrm{GHz}$. This cutoff frequency would be expected to increase as $T^{3 / 2}$, therefore at elevated temperatures, such relaxation might play a rôle with molecular rotational (or perhaps vibrational) relaxation. In one way it is unfortunate that such relaxation is so weak. If electric dipole relaxation were stronger, significant rotational-translational energy exchange might be expected to occur for heteronuclear molecules. Since the temperature of ions can be sensitively monitored by the bolometric technique $[7,8]$, such relaxation might be a way to detect rotational/vibrational transitions in molecular ions. We note that significant rotational (vibrational)-translational energy exchange might be expected to occur between stored molecular ions and a neutral background gas (because of the shorter range of interaction); for this case, the bolometric technique might be used to detect rotational/vibrational transitions in the ions even though part of the energy released in the collision goes to the neutral. 
Relaxation of atomic ions in metastable excited levels (e.g. the lowest ${ }^{2} \mathrm{D}_{5 / 2}$ states in $\mathrm{Ba}^{+}$or $\mathrm{Hg}^{+}$) can proceed via ion-neutral collisions (expected to be efficient) or via the electric fields of ion-ion collisions. For the $\mathrm{D}$ states of $\mathrm{Ba}^{+}$or $\mathrm{Hg}^{+}$, the collisional electric field from ion-ion collisions mixes some ${ }^{2} \mathrm{P}_{3 / 2}$ state into the ${ }^{2} \mathrm{D}_{5 / 2}$ state; therefore during the collision the ion decays at a rate given approximately by $\left[\left\langle{ }^{2} \mathrm{P}_{3 / 2}|q \mathbf{r} \cdot \mathbf{E}|{ }^{2} \mathrm{D}_{5 / 2}\right\rangle /\left(E\left({ }^{2} \mathrm{P}_{3 / 2}\right)-E\left({ }^{2} \mathrm{D}_{5 / 2}\right)\right)\right]^{2} A\left({ }^{2} \mathrm{P}_{3 / 2}\right)$ where $E(\mathrm{i})$ and $A$ (i) are the energy and decay rate of state $i$. If as before, the collision is assumed to have a duration $2 r / v$ then when averaged over impact parameters between $d_{0}$ and $n^{-1 / 3}$, the probability of de-excitation per collision is given by $\langle P\rangle \cong 4 q^{4}$ $a_{0}^{2} n^{2 / 3} A\left({ }^{2} \mathrm{P}_{3 / 2}\right) /\left(\Delta E^{2} d_{0} v\right)$ where $\Delta E \equiv E\left({ }^{2} \mathrm{P}_{3 / 2}\right)-E\left({ }^{2} \mathrm{D}_{5 / 2}\right)$, and we have assumed $\left\langle{ }^{2} \mathbf{P}_{3 / 2}|q \mathbf{r} \cdot \mathbf{E}|{ }^{2} \mathrm{D}_{5 / 2}\right\rangle=q a_{0}|\mathbf{E}|$. Therefore the overall relaxation rate for this process is given by $\langle P\rangle \cdot v n^{1 / 3}$; for $\mathrm{Hg}^{+}$ions at $n=10^{8} / \mathrm{cm}^{3}$ and $T=300 \mathrm{~K}$, the relaxation rate from the ${ }^{2} \mathrm{D}_{5 / 2}$ state is about $5 \times 10^{-9} / \mathrm{s}$ which is negligible.

Therefore we see that at high vacuum, relaxation of the internal energy states of ions is expected to be extremely weak. This may be regarded as an advantage if for example, the objective of an experiment is to obtain sub-Hertz linewidths in double resonance experiments, but may be regarded as a disadvantage if the ions become « trapped " in a metastable state. In this case a buffer gas such as $\mathrm{He}$ or $\mathrm{H}_{2}$ might be added to provide the necessary relaxation [9].

2.2 ENERGY SHIFTS. - One of the primary advantages of the stored ion technique is that ions can be confined in a small region of space without the usual perturbations associated with confinement (e.g. shifts due to ion-neutral collisions in a buffer gas cell).

At finite vacuum one must still worry about ion-neutral collisions but the resulting shifts should be quite small. For example, the fractional shifts of the ground state hyperfine frequencies of ${ }^{137} \mathrm{Ba}^{+}$(Ref. [10]) and ${ }^{199} \mathrm{Hg}^{+}$(Ref. [11]) due to He collisions have been measured to be about $5 \times 10^{-11} / \mathrm{Pa}$ and $4 \times 10^{-11} / \mathrm{Pa}$ respectively. Therefore, at $10^{-8} \mathrm{~Pa}$, such shifts are extremely small. Similarly, on optical transitions, frequency shifts due to ion-neutral collisions would be expected to be smaller than the collision rates; in the high vacuum case fractional shifts would therefore be very small.

One must also consider the frequency shifting effects of the electric fields of ionion collisions and ion-trap " collisions " [12]. The latter effect is analogous to ionneutral collisions in a buffer gas cell however the "collisions" with the electric fields of the ion traps can be much softer. If the ions have linear shifts of energy levels with respect to electric field $(\delta v \propto\langle\mathbf{E}\rangle)$, then these shifts averaged in time must vanish. We know that because if $\langle\mathbf{E}\rangle \neq 0$ the ions would leave the trap.

Energy shifts of non-spherical electronic levels (e.g. the D states in $\mathrm{Ba}^{+}$or $\mathrm{Hg}^{+}$) due to the electric quadrupolar trapping fields of a Penning trap [3, 4] occur at a level of a few $\mathrm{Hz}$ [13]; these shifts should however be precisely controllable. For clouds of many ions in a trap, the ion cloud can also have a quadrupole moment which can interact with an atomic quadrupole moment. Such shifts might become important in very high resolution optical spectra for example. To estimate such an effect, assume that we have a " cold " ion cloud in an if trap. Its shape will be determined by a balance between the forces from the trap pseudopotential and the space charge [3, 4]. If there is no D.C. bias on the ring electrode, the potential from the ions is then given by $\phi$ (ions) $=-\pi q n\left(x^{2}+y^{2}+4 z^{2}\right) / 3$. Using equations (3.6) of Kopfermann [14], the quadrupole energy shift is given by $\Delta E_{\mathrm{Q}}=q\left[Q_{x x} \phi_{x x}+\right.$ $\left.Q_{y y} \phi_{y y}+Q_{z z} \phi_{z z}\right]$ where $Q_{\mathrm{ii}}$ is the ith diagonal element of the atomic quadrupole 
tensor [14] and $\phi_{x x} \equiv \partial^{2} \phi / \partial x^{2}$ etc. Therefore we have approximately $\Delta v_{Q}=\Delta E_{\mathrm{Q}} / h$ $\cong q^{2} n Q / h$ where $Q$ is the usual quadrupole moment [14]. For $Q \cong(1 \AA)^{2}, \Delta v_{\mathrm{Q}} \cong 3$ $\times 10^{-9} n \mathrm{~Hz}$. This shift is probably negligible except perhaps in small $\mathrm{rf}$ traps where very deep pseudo potential wells and high densities can be obtained. One must also worry about quadrupole shifts if collisions are asymmetric; this might be the case if for example, the $x, y$ motion was hotter than the $z$ motion. As an extreme case, assume that two cold ions are trapped in a deep pseudo potential well at a fixed separation of $1 \mu \mathrm{m}$ along the $z$ axis. In this case, $\Delta v_{\mathrm{Q}} \cong q Q \phi_{z z} / h=2 q^{2} Q / h z^{3}$ $\cong 7 \mathrm{kHz}$.

One is often concerned with quadratic Stark shifts $\left(\delta v \propto\left\langle E^{2}\right\rangle\right)$; the source of the electric fields may be due to the trapping fields, ion-ion interactions, optical pumping radiation or perhaps the black body radiation field which at room temperature has a value $[15]\left\langle E^{2}\right\rangle \cong 70(\mathrm{~V} / \mathrm{cm})^{2}$. The shifting effects of optical pumping radiation have been known for quite some time; due to the near resonance condition of this radiation, resulting shifts can be quite large. In high precision double resonance experiments it is usually necessary to probe the transitions with the pumping radiation off (i.e. : chop the pumping light). Electric fields due to trapping $[3,4]$ can be of the order of $100 \mathrm{~V} / \mathrm{cm}$ but in many cases they can be significantly smaller [12]. We can estimate $\left\langle E^{2}\right\rangle$ due to ion-ion collisions by assuming that the electric field is due to binary collisions $\left(E(r)=q / r^{2}\right)$. If we average $E^{2}(r)$ over the volume between two spheres defined by the minimum distance of closest approach and the mean spacing between ions, we obtain $\left\langle E^{2}\right\rangle=3 q^{2} n / d_{0}$. For $T=300 \mathrm{~K}$, $n=10^{8} / \mathrm{cm}^{3},\left\langle E^{2}\right\rangle \cong 3(\mathrm{~V} / \mathrm{cm})^{2}$.

For hyperfine structure, frequency shifts due to quadratic Stark effects can be quite small. For example, for $\mathrm{Ba}^{+}$ground state hyperfine structure $\left(\delta v / v_{0}\right) \cong 3.5$ $\times 10^{-17}\left\langle E^{2}\right\rangle$ where $E$ is expressed in $\mathrm{V} / \mathrm{cm}$ [15]. For optical structure, we can for example estimate the shift of the ${ }^{2} \mathrm{D}_{5 / 2}$ state in $\mathrm{Hg}^{+}$or $\mathrm{Ba}^{+}$as $\delta v=\mid\left\langle{ }^{2} \mathrm{P}_{3 / 2}\right.$ $\left.|q \mathbf{r} \cdot \mathbf{E}|{ }^{2} \mathrm{D}_{5 / 2}\right\rangle\left.\right|^{2} / h\left(E\left({ }^{2} \mathrm{P}_{3 / 2}\right)-E\left({ }^{2} \mathrm{D}_{5 / 2}\right)\right)=q^{2} a_{0}^{2}\left\langle E^{2}\right\rangle / \Delta E h$ using the same approximation as previously. For $\mathrm{Hg}^{+}, \delta v \cong\left\langle E^{2}(\mathrm{~V} / \mathrm{cm})\right\rangle .(0.002 \mathrm{~Hz})$. Therefore quadratic Stark shifts can be quite small; they can be further reduced by chopping the pumping light, cooling the kinetic temperature of the ions [12] (see below) and reducing the ambient temperature to reduce the black-body radiation fields.

For ions stored in a Penning trap, the required magnetic fields are on the order of 1 tesla. In this case, therefore, shifts of atomic ion energy levels can be quite strong. Of course the study of magnetic interactions appears to be quite interesting, and certain effects such as diamagnetic shifts to atomic hyperfine structure have been only recently measured [16]. For high resolution double resonance experiments, magnetic field dependent shifts could be regarded as a severe disadvantage because of field fluctuations, but in certain cases, transition frequencies become immune to field fluctuations to first order and extremely high resolution can be obtained [17-19].

2.3 Ion NUMBER. - Typically, the numbers of ions in stored ion experiments are rather small. Densities are on the order of $10^{6} / \mathrm{cm}^{3}$ and sample sizes are $\lesssim 1 \mathrm{~cm}^{3}$. For many experiments (such as spectroscopy of molecular ions), this is a disadvantage but in some cases even single ions can be sensitively detected [20-22]. The small densities obtained are to be compared with the much larger densities in buffer gas experiments [2] where space charge neutralization is used. In rf traps, some space charge neutralization has been realized $[23,24]$ by storing ions of opposite charge but this technique has not been widely used perhaps because of the mechanism of of heating $[7,25]$. 
Although the ion numbers are typically rather small, usable samples can be confined to an extremely small region of space - down to less than $(1 \mu \mathrm{m})^{3}$ for the case of single ions $[20,22]$. This is important because in certain high resolution experiments on optical spectra, it is desirable to satisfy the Lamb-Dicke criterion (confinement to dimensions less than $\lambda / 2 \pi$ ) in order to suppress first order Doppler broadening [13]. Small sample sizes also reduce the requirements on magnetic field homogeneity in high resolution magnetic field dependent spectra.

\section{Direct optical pumping.}

The essential features of optical pumping in ion traps are not different from the experiments on neutrals or ions in buffer gas cells. Nevertheless, there are some unique features of optical pumping on trapped ions; here we give a simple example.

In the inset in figure 1 , we show the $3 s^{2} \mathrm{~S}_{1 / 2}$ and $3 p^{2} \mathrm{P}_{3 / 2}$ level structure of ${ }^{24} \mathrm{Mg}^{+}$ ions in a magnetic field. If a laser (assumed to have linear polarization perpendicular to the magnetic field) is tuned to the $(-1 / 2) \rightarrow(-3 / 2)$ transition (numbers in parentheses refer to the ground ${ }^{2} \mathrm{~S}_{1 / 2}$ and excited ${ }^{2} \mathrm{P}_{3 / 2}$ values of $M_{3}$ respectively) then we note that ions must decay to the $M_{\mathrm{J}}=-1 / 2$ ground level because of the selection rule $\Delta M_{\mathrm{J}}=0, \pm 1$. At first, one might guess that after many photon scattering events, the ions are gradually pumped from the $M_{\mathrm{J}}=-1 / 2$ to $M_{\mathrm{J}}=+1 / 2$ ground state level because of excitation in the wings of other allowed transitions. In fact just the opposite occurs. Although excitation in the wings of the $(-1 / 2) \rightarrow$ $(+1 / 2)$ transition pumps ions out of the $(-1 / 2)$ ground state, excitation in the

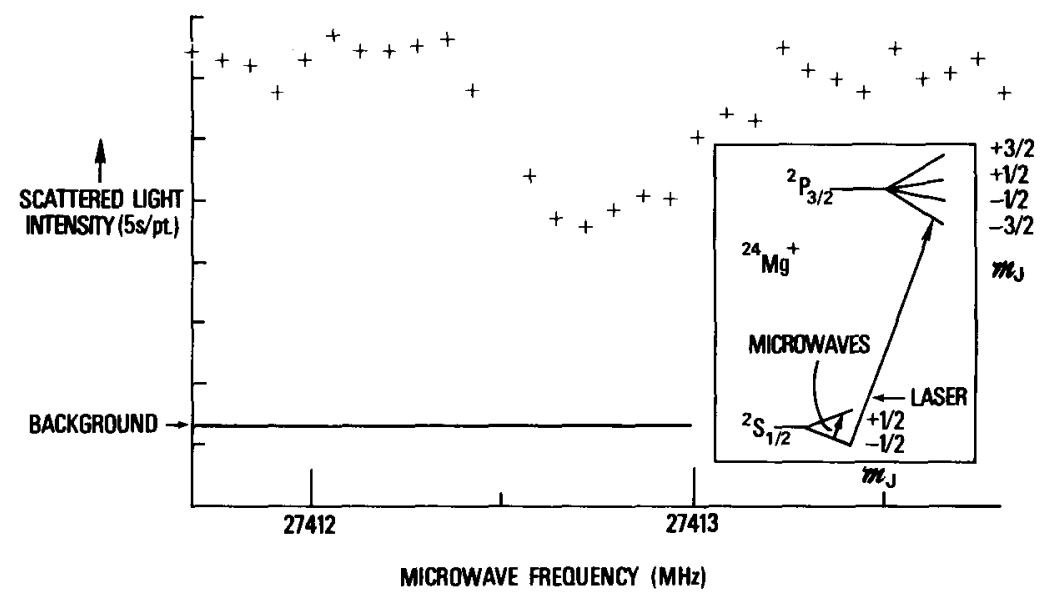

Fig. 1. - Microwave-optical double resonance spectrum of ${ }^{24} \mathrm{Mg}^{+}$. Inset shows relevant energy levels of ${ }^{24} \mathrm{Mg}^{+}$in a magnetic field. With the laser tuned to the transition shown, $16 / 17$ of the ions are pumped into the ${ }^{2} S_{1 / 2}\left(M_{\mathrm{J}}=-1 / 2\right)$ ground state and a quasi two level system is formed with this ground state and the excited ${ }^{2} \mathbf{P}_{3 / 2}\left(M_{\mathrm{J}}=-3 / 2\right)$ state. When incident microwaves are tuned to the $\left(M_{\mathrm{J}}=-1 / 2\right) \leftrightarrow\left(M_{\mathrm{J}}=+1 / 2\right)$ ground state Zeeman transition, these levels are nearly equally populated which causes a decrease in fluorescence scattering from the ions. Transitions in other ions can be detected in a similar way (from Ref. [26]).

[Spectre de double résonance micro-onde-optique de ${ }^{24} \mathrm{Mg}^{+}$. L'encadré montre les niveaux d'énergie concernés de ${ }^{24} \mathrm{Mg}^{+}$dans un champ magnétique.] 
wings of the $(+1 / 2) \rightarrow(-1 / 2)$ transition also occurs which tends to pump ions from the $(+1 / 2)$ to $(-1 / 2)$ ground state. Since this latter transition is four times closer in frequency to the laser than the former one, the net result is that about $16 / 17$ of the population is pumped into the $(-1 / 2)$ ground state [26]. Of course, by using circularly polarized light, all of the population could be pumped to the $(-1 / 2)$ ground state. This pumping is very weak (the time constant for pumping in this experiment was a few seconds) but because of the very long relaxation times of the ions in the trap it can be very efficient.

This experiment is somewhat unusual because, using linearly polarized light, the ions are pumped into the ground state energy level that the laser is tuned to. However, noting that the $(-1 / 2) \rightarrow(-3 / 2)$ transition takes no part in the pumping, this is just another case of depopulation pumping (out of the $(+1 / 2)$ ground state) using frequency selected light [27]. Similar optical pumping can also be observed when hyperfine structure is present ; for example [18], in the case of ${ }^{25} \mathrm{Mg}^{+}$and ${ }^{9} \mathrm{Be}^{+}$. Hyperfine pumping effects have also been observed [28] in the excited ${ }^{3} \mathrm{~S}_{1}$ states of $\mathrm{Li}^{+}$.

Depopulation pumping in the more typical sense (i.e. depopulation of the ground state level one is driving on) occurs if one excites for example the ${ }^{2} \mathrm{~S}_{1 / 2} \rightarrow{ }^{2} \mathrm{P}_{1 / 2}$ transitions in alkali-like ions. In this case one must redistribute or mix the ground state populations in order to see additional scattering from the pumping source. In this instance one of the advantages of the traps for high resolution spectroscopy (weak relaxation) becomes a disadvantage in terms of observation. Solutions to this problem are : (1) Provide a buffer gas for relaxation. For example, Ruster et al. [9] observed fluorescence from single $\mathrm{Ba}^{+}$ions in an $\mathrm{rf}$ trap by relaxing the ions against an $\mathrm{H}_{2}$ buffer gas. (2) If the number of ground (and metastable) states is not too large, artificial relaxation can be provided by auxiliary microwave or laser radiation $[4,20,29,30]$.

\section{Optical pumping-double resonance.}

Double resonance detection of atomic ion spectra using traps is achieved in most cases using the same techniques developed for neutrals. However, because of the weak relaxation and high scatter rates using lasers, some novel effects appear. For example, in the ${ }^{24} \mathrm{Mg}^{+}$case above, if the scattered fluorescence light from the ions is monitored, the $\left(M_{\mathrm{J}}=-1 / 2\right)$ to $\left(M_{\mathrm{J}}=+1 / 2\right)$ ground state Zeeman transition (induced by microwave radiation) can be detected by observing the decrease in laser fluorescence as the microwave oscillator is swept through resonance (Fig. 1). An interesting feature of this optical pumping-double resonance detection scheme is that each microwave photon absorbed causes a change of about $\Delta N^{*}=24 B(-1 / 2$, $-3 / 2) / 17 B(-1 / 2,+1 / 2)$ in the number of scattered photons where $B\left(M_{\mathrm{J}}, M_{\mathrm{j}}^{\prime}\right)$ is the transition rate due to the laser from the $M_{\mathrm{J}}$ ground state to the $M_{\mathrm{J}}^{\prime}$ excited state. For $\mathrm{Mg}^{+}$ions in a magnetic field of about $1 \mathrm{~T}, \Delta N^{*}$ can be as high as $2 \times 10^{6}$. This technique has been called « electron shelving " because the ion's outer electron is temporarily "shelved" in a state from which the laser scattering is essentially absent [31]. It has been used in all the optical pumping double resonance experiments on $\mathrm{Mg}^{+}$and $\mathrm{Be}^{+}$ions stored in Penning traps. This photon amplification scheme was independently developed in optical pumping experiments on neutral atomic beams [32].

The overall detection sensitivity is increased by this photon number amplification effect and also by the photon energy conversion. Perhaps noteworthy is the case of 
detecting absorbed $303 \mathrm{MHz}$ photons in $\mathrm{Be}^{+}$hyperfine transitions [33] where an energy enhancement factor of $\Delta N^{*} \lambda(303 \mathrm{MHz}) / \lambda$ (laser) $>10^{12}$ is obtained. These impressive numbers are of course not realized in practice since fluorescence collection efficiencies are typically significantly less than $100 \%$. However, a more important statement is that if (the absence of) enough scattered photons per ion (theoretically $\gtrsim 2$ ) are observed before repumping takes place, then the signal to noise ratio in such experiments need only be limited by the statistical fluctuations in the number of ions that make the transition [34]. This is the maximum signal to noise ratio possible.

The long relaxation times and small perturbations achieved in the traps can lead to very high resolution and accuracy. In ${ }^{25} \mathrm{Mg}^{+}$, the first derivative of the ground state $\left(M_{\mathrm{I}}=-3 / 2, M_{\mathrm{J}}=1 / 2\right)$ to $\left(M_{\mathrm{I}}=-1 / 2, M_{\mathrm{J}}=1 / 2\right)$ transition goes to zero at a value of the magnetic field near $1.24 \mathrm{~T}$. Near this field, a resonance with a width of $0.012 \mathrm{~Hz}$ and a center frequency of 291.996251899 (3) $\mathrm{MHz}$ was observed (Fig. 2) [18]. High resolutions have also been obtained in $\mathrm{Yb}^{+}\left(\Delta v \cong 0.06 \mathrm{~Hz}, Q \cong 2 \times 10^{11}\right.$ [35]) and $\mathrm{Hg}^{+}\left(\Delta v=0.85 \mathrm{~Hz}, Q \cong 5 \times 10^{10}\right.$ [11]). Very high accuracies in stored ion spectra have also been achieved $[11,30,33,34]$. In the case of $\mathrm{Be}^{+}$, the $\left(M_{1}, M_{\mathrm{j}}\right)$ $=(-3 / 2,1 / 2) \rightarrow(-1 / 2,1 / 2)$ ground state hyperfine transition frequency at the magnetic field independent point has been measured with an accuracy of about one part in $10^{13}$ [33]. From the discussion of section 2, it should be possible to obtain much higher resolutions and accuracies.

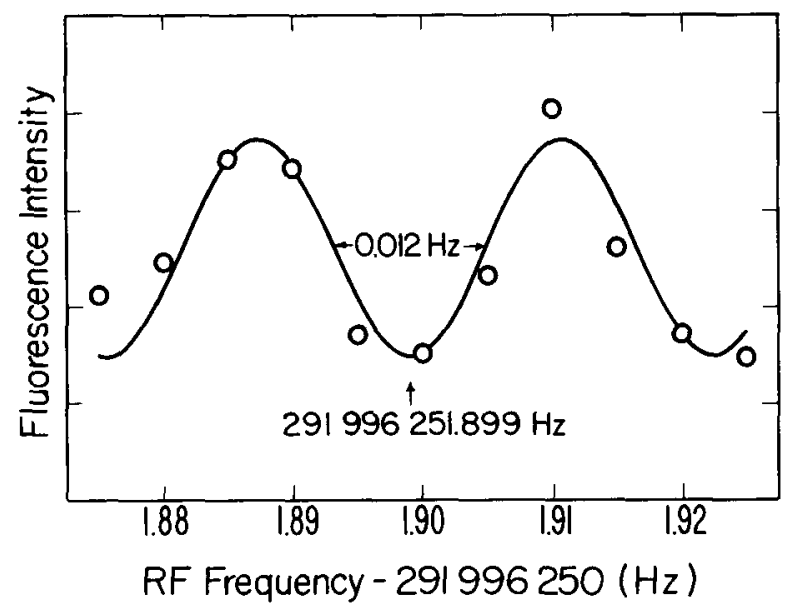

Fig. 2. - The $\left(M_{1}=-3 / 2, M_{\mathrm{J}}=1 / 2\right)$ to $\left(M_{\mathrm{I}}=-1 / 2, M_{\mathrm{J}}=1 / 2\right)$ resonance in ${ }^{25} \mathrm{Mg}^{+}$ about $1.24 \mathrm{~T}$. The oscillatory lineshape results from the use of the Ramsey separated oscillatory field method, implemented by applying two phase-coherent RF pulses $1.02 \mathrm{~s}$ long, separated by $41.4 \mathrm{~s}$ (from Ref. [18]).

[Résonance $\left(M_{\mathrm{I}}=-3 / 2, M_{\mathrm{J}}=1 / 2\right) \rightarrow\left(M_{\mathrm{I}}=-1 / 2, M_{\mathrm{J}}=1 / 2\right)$ dans ${ }^{25} \mathrm{Mg}^{+}$à environ $1,24 \mathrm{~T}$. La forme de raie oscillante résulte de l'utilisation de la méthode de Ramsey à champs oscillants séparés; on applique ici deux impulsions RF cohérentes en phase de durée 1,02 s, séparées par 41,4 s (Réf. [18]).] 


\section{Optical pumping of kinetic energy levels-laser cooling.}

In 1950, Kastler proposed the idea of «lumino refrigeration" [1]. His idea was to cool the salts of the rare earth ions with an anti-Stokes transition of sufficient intensity. The basic principle was that perhaps ions embedded in a solid could be made to undergo anti-Stokes scattering where the energy deficit would be supplied by the energy of lattice vibrations. Partly because of different motivations (high resolution spectroscopy) and different technology available (the laser) this idea was reinvented for atoms [36] and trapped ions [37] in 1975.

Laser cooling of trapped ions can be described in various ways [29], here we briefly discuss the analogy to optical pumping and anti-Stokes scattering [38]. In figure 3 we have illustrated the energy levels of a trapped ion which we assume has an internal structure composed of two states - the ground (g) and excited (e) states. If we assume that the ion is also constrained to move along one direction in a harmonic well (frequency $v_{\mathrm{v}}$ ) then its kinetic energy levels are given by $E_{\mathrm{K}}=(n+1 / 2) h v_{\mathrm{v}}$. In this picture we can think of the ion bound to the trap as a kind of « super-molecule » whose energy level structure can be investigated [38].

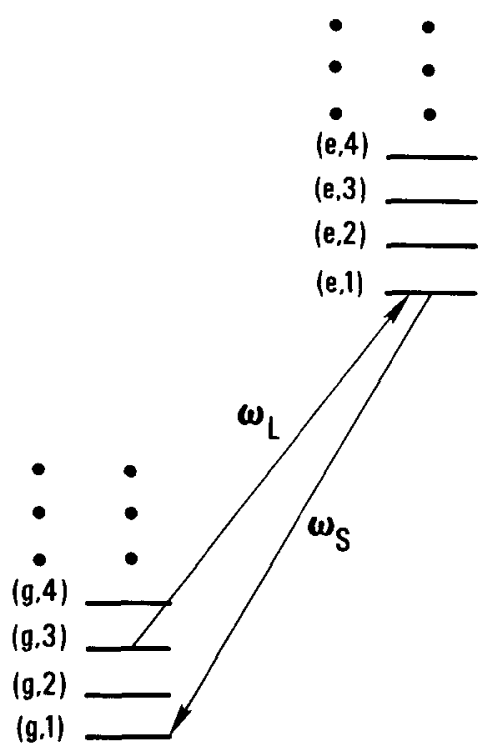

Fig. 3. - Pictorial representation of electronic [ground (g) and excited (e)] energy states and translational energy states (denoted by integers) for an atom. When the atom is very weakly bound or unbound the spacing between translational energy levels goes to zero. The cooling can be described as anti-Stokes Raman scattering or optical pumping where the frequency $\left(\omega_{\mathrm{s}}\right)$ of the scattered photon is greater than the frequency $\left(\omega_{\mathbf{L}}\right)$ of the "laser " photon (from Ref. [38]).

[Schéma des niveaux d'énergie électroniques (fondamental ( $\mathrm{g}$ ) et excité (e)) et de translation (repérés par des entiers) pour un atome. Quand l'atome est très faiblement lié, ou libre, la séparation entre niveaux d'énergie de translation tend vers zéro. Le refroidissement peut être décrit comme une diffusion Raman anti-Stokes, ou un pompage optique où la fréquence $\omega_{\mathrm{S}}$ du photon diffusé est plus grande que la fréquence $\omega_{\mathrm{L}}$ du photon « laser » (Réf. [38]).] 
Assume the ion is initially in the $\left(\mathrm{g}, n_{\mathrm{i}}\right)$ state (normally we would expect it to be in some distribution of states). Then if we excite the $\left(\mathrm{g}, n_{\mathrm{i}}\right) \rightarrow\left(\mathrm{e}, n_{\mathrm{i}}-\Delta n\right)$ transition with radiation, the ion can decay to a number of lower states but (neglecting terms of order $\left.R=h^{2} / 2, \lambda^{2} m\right)$ on the average it decays to the ( $\left.\mathrm{g}, n_{\mathrm{i}}-\Delta n\right)$ ground state. Therefore in a single scattering event, the ion's kinetic energy has been reduced by $h v_{v} \Delta n$. Laser cooling of free atoms can also be described in this picture; in this case $v_{v} \rightarrow 0$ and the ground and excited states become continuous bands. In principle, laser cooling should work for solids but inhomogeneous broadening and non radiative relaxation would appear to cause problems.

Laser cooling of trapped ions has been accomplished in experiments at the National Bureau of Standards, Heidelberg and Hamburg, University of Washington and at Orsay $[4,12,13,18-22,25,29-31,39]$. Temperatures of $<1 \mathrm{~K}$ are not uncommon for a small sample of ions; these low temperatures significantly reduce Doppler effects in those experiments.

\section{Exchange pumping.}

6.1 EXCHANGe PUMPING OF INTERNAL ENERGY LEVELS. - Although polarization or pumping of trapped ions using polarized, externally injected neutral beams has been accomplished [3, 40,41], exchange pumping due to ion-ion collisions would ordinarily seem to be precluded. This is illustrated by the weak relaxation discussed in section 2.1 and is mainly due to the fact that the Coulomb repulsion usually prevents the ions from getting close enough for exchange to occur. For room temperature ions, $d_{0} \cong 200 \AA$. At elevated temperatures, exchange pumping might be expected to take place; for ions of energy $10 \mathrm{eV}, d_{0} \lesssim 1 \AA$. If it could be realized, such exchange pumping might significantly extend spectroscopic measurements on ions as it has for the case of neutral-neutral and ion-neutral collisions.

6.2 EXCHANGe PUMPING OF KINETIC ENERGY LEVELS. - In the sense that an ion bound in the trap forms a supermolecule as in section 5, we can think of performing exchange pumping on the kinetic energy levels of the ions. The idea of buffer gas cooling of ions $[3,25,29,30,42-51]$ fits within this context. Here the (bound) ions exchange their kinetic energy with a light buffer gas (e.g. He, or $\mathrm{H}_{2}$ ) whose kinetic energy is thermalized (or " pumped » in the sense here) to the ambient temperature. Analogous arguments could be made for radiative damping of the ions $[7,8]$.

Although relaxation between the internal states of ions due to ion-ion collisions is rather weak (except at elevated temperatures) the relaxation between kinetic degrees of freedom is rather strong due to the long range of the Coulomb interaction. At temperatures near room temperature, this relaxation time is typically of order $10 \mathrm{~ms}$. (See for example Refs. [3, 7, 8, 25, 52]). Such collisional coupling between different degrees of freedom can be important in the laser cooling experiments when only one or two kinetic degrees of freedom are directly cooled by the laser and the remaining degree(s) of freedom are heated by photon recoil [38, 53].

This coupling may also extend the number of ion species which can benefit from laser cooling. That is, we could simultaneously store two kinds of ions, one which is easily laser cooled and another one which is of spectroscopic interest and via the Coulomb collisions is also cooled [54]. This has been demonstrated in experiments on $\mathrm{Mg}^{+}$where ${ }^{24} \mathrm{Mg}^{+}$was laser cooled and by collisions " sympathetically " cooled ${ }^{25} \mathrm{Mg}^{+}$and ${ }^{26} \mathrm{Mg}^{+}$[55]. We note that in subsequent experiments at NBS it was possible to sympathetically cool ${ }^{24} \mathrm{Mg}^{+}$by laser cooling ${ }^{25} \mathrm{Mg}^{+}$; this result 
is more definitive because the laser was tuned to the heating side of the ${ }^{24} \mathrm{Mg}^{+}$ transition.

It is interesting to note that at very low temperature (the case for a laser cooled cloud of ions) we can expect species of ions with different charge to mass ratio to centrifugally separate [56]. This can be seen by considering two ion species ( 1 and 2) of different charge to mass ratio, which are in thermal equilibrium in a Penning trap. In this case we can write the distribution function for the ions as a product [56] :

where

$$
\begin{gathered}
f=f_{1} f_{2} \\
f_{\mathrm{j}}=n_{\mathrm{j}}(0)\left(\frac{m_{\mathrm{j}}}{2 \pi k_{\mathrm{B}} T}\right)^{3 / 2} \exp \left[-\frac{\left(H_{\mathrm{j}}+\omega P_{\theta \mathbf{j}}\right)}{k_{\mathrm{B}} T}\right] \\
=n_{\mathrm{j}}(\mathbf{r})\left(\frac{m_{\mathrm{j}}}{2 \pi k_{\mathrm{B}} T}\right)^{3 / 2} \exp \left[-\frac{m_{\mathrm{j}}}{2 k_{\mathrm{B}} T}(\mathbf{v}+\omega \hat{\mathbf{\theta}})^{2}\right] \\
n_{\mathrm{j}}(\mathbf{r})=n_{\mathrm{j}}(\mathbf{r}=0) \mathrm{e}^{-\psi_{\mathrm{j}}(\mathbf{r})} \\
\psi_{\mathrm{j}}(\mathbf{r})=\frac{q_{\mathrm{j}}}{k_{\mathrm{B}} T}\left(\phi(\mathbf{r})-\frac{m_{\mathrm{j}}}{q_{\mathrm{j}}} \frac{\omega^{2} r^{2}}{2}+\frac{B \omega r^{2}}{2 c}\right)
\end{gathered}
$$

where

$$
\phi(\mathbf{r})=\phi(x, y, z)=\phi(r, z)=\phi(\text { ions })+\phi(\text { trap }),
$$

$r$ and $z$ are cylindrical coordinates, and the (conserved) angular momentum $\left(P_{\theta \mathrm{j}}\right)$ is parallel to the $z$ axis of the trap and the magnetic field $\mathbf{B}=B \hat{\mathbf{z}}$. The ion cloud behaves as a rigid rotor plasma [57] which rotates at frequency $-\omega$. For the distribution function to be well behaved as $T \rightarrow 0$, we must have

$$
\phi(\mathbf{r})-\frac{m_{\mathrm{j}}}{q_{\mathrm{j}}} \frac{\omega^{2} r^{2}}{2}+\frac{B \omega r^{2}}{2 c} \rightarrow 0
$$

therefore the charge density inside the cloud is given by [53]

$$
\rho=-\frac{1}{4 \pi} \nabla^{2} \phi \text { (ions) }=\frac{1}{2 \pi}\left(\frac{B \omega}{c}-\frac{2 m_{\mathbf{j}}}{q_{\mathrm{j}}} \omega^{2}\right)
$$

where we have used the fact that $\nabla^{2} \phi($ trap $)=0$. For the density to be single valued, ions with different $m_{\mathrm{j}} / q_{\mathrm{j}}$ cannot overlap. For the case of a very weak axial well, the cloud breaks up into a cylinder of uniform density $\rho_{1}$ surrounded by an annulus of uniform density $\rho_{2}$ [56]. (We have assumed $m_{1} / q_{1}<m_{2} / q_{2}$.) Solutions in the more typical case will be discussed in a future publication. A similar separation is expected to occur in an if trap except when there is no angular momentum in the cloud. We note that in an experiment using lasers, we would, in general expect there to be angular momentum in the cloud unless the laser beam exactly overlaps the center of the cloud. Qualitatively, the separation should occur because for a given total angular momentum, a friction force exists between species of different $m / q$; ions of lower $m / q$ tend to push out ions of higher $m / q$ and vice versa.

As a potential application, spectroscopy could be done on ${ }^{9} \mathrm{Be}^{+}$ions which are held at the center of the trap and sympathetically cooled by an outer annulus of ${ }^{24} \mathrm{Mg}^{+}$ions which are laser cooled. The cooling laser could be applied so that it would not spatially overlap the ${ }^{9} \mathrm{Be}^{+}$ions and therefore light shifts on the ${ }^{9} \mathrm{Be}^{+}$ 
energy levels could be avoided. In this way, extremely narrow linewidths ( $\ll 1 \mathrm{mHz})$ and high accuracy spectra on $\mathrm{Be}^{+}$(or other ions) might be obtained. For ions in an if trap, sympathetic cooling may be limited by of heating.

\section{Acknowledgments.}

The authors greatfully acknowledge the support of the U.S. Air Force Office of Scientific Research and the U.S. Office of Naval Research. We thank P. L. Bender and F. L. Walls for reading the manuscript and for helpful comments.

\section{References}

[1] Kastler, A., J. Phys. Rad. 11 (1950) 255.

[2] Weber, E. W., Phys. Rep. 32 (1977) 123.

[3] Dehmelt, H. G., Adv. At. Mol. Phys. 3 (1967) 53 and Adv. At. Mol. Phys. 5 (1969) 109.

[4] Wineland, D. J., Itano, W. M. and Van Dyck, Jr., R. S., Adv. At. Mol. Phys. 19 (1983) 135 and references therein.

[5] $133 \mathrm{~Pa}$ (Pascals) $\cong 1$ torr.

[6] See for example : E. W. MCDAniel, Collision Phenomenon in Ionized Gases, Wiley, New York, 1964.

[7] Church, D. A. and Dehmelt, H. G., J. Appl. Phys. 40 (1969) 3421.

[8] Dehmelt, H. G. and Walls, F. L., Phys. Rev. Lett. 21 (1968) 127 ; Wineland, D. J. and Dehmelt, H. G., J. Appl. Phys. 46 (1975) 919.

[9] Ruster, W., Bonn, J., Peuser, P. and Trautmann, N., Appl. Phys. B 30 (1983) 83; Blatt, R. and Werth, G., Phys. Rev. A 25 (1982) 1476.

[10] Vetter, J., Stuke, M. and Weber, E. W., Z. Physik A 273 (1975) 129.

[11] Cutler, L. S., Giffard, R. P. and McGuire, M. D., Appl. Phys. B 36 (1985) 137.

[12] Wineland, D. J., in « Precision Measurement and Fundamental Constants II " (B. N. Taylor and W. D. Phillips, eds.). NBS Spec. Publ. (U.S.) 617 (1984) 83.

[13] Dehmelt, H., IEEE Trans. Instrum. Meas. IM-31, 83 (1982).

[14] Kopfermann, H., Nuclear Moments (Academic, New York, 1958), p. 11.

[15] Itano, W. M., Lewis, L. L., Wineland, D. J., Phys. Rev. A 251233 (1982).

[16] Economou, N. P., Lipson, S. J. and Larson, D. J., Phys. Rev. Lett. 38 (1977) 1394.

[17] Kusch, P. and TaUb, H., Phys. Rev. 75 (1949) 1477.

[18] Itano, W. M. and Wineland, D. J., Phys. Rev. A 24 (1981) 1364.

[19] Bollinger, J. J., Itano, W. M. and Wineland, D. J., Phys. Rev. Lett. 54 (1985) 1000.

[20] Neuhauser, W., Hohenstatt, M., Toschek, P. and Dehmelt, H. G., Phys. Rev. A 22 (1980) 1137.

[21] Wineland, D. J. and Itano, W. M., Phys. Lett. 82A (1981) 75.

[22] Nagourney, W., JaniK, G. and Dehmelt, H., Proc. Natl. Acad. Sci. USA 80 (1983) 643.

[23] Major, F. G. and Schermann, J. P., Bull. Am. Phys. Soc. 16 (1971) 838.

[24] AUdoIn, C., private communication.

[25] Dehmelr, H. G., in "Advances in Laser Spectroscopy" (F. T. Arecchi, F. Strumia and H. Walther, Eds.). Plenum, New York (1983) p. 153.

[26] Wineland, D. J., Bergquist, J. C., Itano, W. M. and Drullinger, R. E., Opt. Lett. 5 (1980) 245.

[27] Happer, W., Rev. Mod. Phys. 44 (1972) 169.

[28] PRIOR, M. H. and KNIGHT, R. D., Opt. Commun. 35 (1980) 54.

[29] Wineland, D. J., Itano, W. M., Bergquist, J. C., Bollinger, J. J. and Prestage, J. D., in Atomic Physics 9, ed. by R. S. Van Dyck, Jr. and E. N. Fortson (World Scientific Publ. Co., Singapore 1985) p. 3. 
[30] WeRth, G., ibid p. 28.

[31] Dehmelt, H. G., J. Physique 42 (1981) C8-299;

Dehmel, H. G., Bull. Am. Phys. Soc. 18 (1973) 1521.

[32] PICQuE, J. L., Metrologia 13 (1977) 115.

[33] Bollinger, J. J., Itano, W. M. and Wineland, D. J., Proc. 37th Annu. Symp. Freq. Control, 37 (1983). (Copies available from Systematics General Corp., Brinley Plaza, Rt. 38 Wall Township, NJ 07719.) J. J. Bollinger, J. D. Prestage, W. M. Itano and D. J. Wineland, Phys. Rev. Lett. 54 (1985) 1000.

[34] Wineland, D. J., Itano, W. M., Bergquist, J. C. and Walls, F. L., Proc. 35th Annu. Symp. Freq. Control (1981) p. 602 (copies available from Electronic Industries Assoc., 2001 Eye St., NW, Washington, DC 20006).

[35] Blatt, R., Schnatz, H. and Werth, G., Phys. Rev. Lett. 48 (1982) 1601.

[36] Hänsch, T. W. and SChawlow, A. L., Opt. Commun. 13 (1975) 68.

[37] Wineland, D. J. and Dehmelt, H. G., Bull. Am. Phys. Soc. 20 (1975) 637.

[38] Wineland, D. J. and Itano, W. M., Phys. Rev, A 20 (1979) 1521 ; Itano, W. M. and Wineland, D. J., Phys. Rev. A 25 (1982) 35.

[39] Plumelle, F., Abstract for 15th EGAS Conf. Madrid, July 5-8 (1983).

[40] Schuessler, H. A., in "Physics of Atoms and Molecules, Progress in Atomic Spectroscopy » (W. Hanle and H. Kleinpoppen, eds.), Plenum, New York, 999 (1979).

[41] Gräff, G., Major, F. G., Roeder, R. W. H. and Werth, G., Phys. Rev. Lett. 21 (1968) 340 ;

GräFf, G., Huber, K., Kalinowsky, H. and Wolf, H., Phys. Lett. 41A (1972) 277 ; GräFF, G. and HolzscheIter, M., Phys. Lett. 79A (1980) 380.

[42] Jardino, M., Plumelle, F., Desaintfuscien, M. and Duchêne, J. L., Proc. 38th Annu. Symp. Freq. Control, Phil. PA (1984) to be published.

[43] Vedel, M., J. Physique 37 (1976) L-336.

[44] Dawson, P. H., Adv. Electron. Electron Phys. Suppl. 13B (1980) 173.

[45] Plumelle, F., Desaintfuscien, M., Duchêne, J. J. and Audoin, C., Opt. Commun. 34 (1980) 71 .

[46] Schaaf, H., Schmeling, U. and Werth, G., Appl. Phys. 25 (1981) 249.

[47] ANDré, J. and Vedel, F., J. Physique 38 (1977) 1381.

[48] ANdré, J., Vedel, F. and Vedel, M., J. Physique 40 (1979) L-633.

[49] Vedel, F., André, J. and Vedel, M., J. Physique 42 (1981) 1611.

[50] Vedel, F., André, J., Vedel, M. and Brincourt, G., Phys. Rev. A 27 (1983) 2321.

[51] Vedel, M., André, J., Chaillat-Negrel, S. and Vedel, F., J. Physique 42 (1981) 541.

[52] Heppner, R. A., Walls, F. L., Armstrong, W. T. and Dunn, G. H., Phys. Rev. A 13 (1976) 1000.

[53] Bollinger, J. J. and Wineland, D. J., Phys. Rev. Lett. 53 (1984) 348.

[54] Wineland, D. J., Drullinger, R. E. and Walls, F. L., Phys. Rev. Lett. 40 (1978) 1639.

[55] Drullinger, R. E., Wineland, D. J. and Bergquist, J. C., Appl. Phys. 22 (1980) 365.

[56] O’NeIL, T. M., Phys. Fluids 24 (1981) 1447.

[57] Davidson, R. C., Theory of Nonneutral Plasmas (Benjamin, Reading MA, 1974). 